\title{
A New Multistatic FMCW Radar Architecture By Over-The-Air Deramping
}

\author{
M. Ash, M. Ritchie, K. Chetty, and P. V. Brennan
}

\begin{abstract}
Frequency modulated continuous wave (FMCW) radar is widely adopted solution for low-cost, short to medium range sensing applications. However, a multistatic FMCW architecture suitable for meeting the low-cost requirement has yet to be developed. This paper introduces a new FMCW radar architecture that implements a novel technique of synchronising nodes in a multistatic system, known as over-the-air deramping (OTAD). The architecture uses a dual-frequency design to simultaneously broadcast an FMCW waveform on a lower frequency channel directly to a receiver as a reference synchronisation signal, and a higher frequency channel to illuminate the measurement scene. The target echo is deramped in hardware with the synchronisation signal. OTAD allows for low-cost multistatic systems with fine range-resolution, and low peak power and sampling rate requirements. Furthermore, the approach avoids problems with direct signal interference. OTAD is shown to be a compelling solution for low-cost multistatic radar systems through experimental measurements using a newly developed OTAD radar system.
\end{abstract}

Index Terms-FMCW radar; bistatic radar; multistatic radar; passive radar; passive bistatic radar; micro-Doppler; distributed sensors.

\section{INTRODUCTION}

Frequency modulated continuous wave (FMCW) radar has emerged as a widely adopted solution for low-cost systems in contemporary literature. It has many useful characteristics such as fine range resolution, good immunity to blocker/interference signals, and low peak power and sampling rate requirements [1]. Applications have included autonomous cruise control systems [2], the measurement of geophysical phenomena [3] and weather radar [4]. However, these systems are all based on a familiar FMCW radar architecture, which only allows for monostatic or wired multistatic measurements.

Multistatic radar systems have many advantages over monostatic systems. In particular, the prospect of improving sensitivity in certain geometric configurations [5], and achieving multi-aspect views of targets, which may enhance target classification [6], make them ideally suited to surveillance and security applications. Furthermore, they are less susceptible to trihedral-like clutter, which is a key advantage over monostatic systems when operating in urban or indoor environments.

There are several types of multistatic radar ranging from complex fully coherent time-synchronised multistatic systems

This work was supported by UK Engineering and Physical Sciences Research Council Bridging the Gaps grant.

M. Ash, M. Ritchie and P. V. Brennan are with the Department of Electronic and Electrical Engineering, University College London, London, UK, WC1E 7JE. e-mail: \{m.ash, m.ritchie, p.brennan\}@ucl.ac.uk.

$\mathrm{K}$. Chetty is with the Department of Security and Crime Science, University College London, London, UK, WC1H 9EZ. e-mail: k. chetty@ucl.ac.uk. to low-cost passive bistatic radar (PBR) systems [5]. Both of these types have their advantages and disadvantages. The former allows for, not only the aforementioned multistatic radar capability, but also target localisation with a resolution beyond that expected from the radar signal bandwidth [7]. However, such systems require the use of expensive and complex methods in order to phase-lock each multistatic node to achieve coherence [8].

Passive bistatic radar utilises illuminators of opportunity, such as radio broadcast stations [9], as a means of measuring a scene. A passive radar node records the signal arriving over a direct path from the broadcast station to the radar and, simultaneously, an echo signal arriving following scattering from the measurement scene. These two signals are then crosscorrelated using digital signal processing to produce range and radial velocity measurements of targets within the scene [10]. Many of these nodes can be deployed around the region illuminated by the broadcast station to widen coverage.

PBR systems have the advantage of being purely passive, and hence covert, and relatively low-cost to deploy. However, they often suffer from coarse range-resolution, depending on the bandwidth of the signal of opportunity, and in reality it is a complex problem to separate the reference signal from the scattered signal. Indeed, the performance of a PBR system is often limited by the signal to direct-interference ratio (SIR) [11]. The SIR can be improved through signal processing [10], [12] or forming antenna nulls in the direction of the broadcast station on the scattered signal channel [13]. However, the complexity of these approaches can start to diminish the lowcost argument for the system. Furthermore, since the system requires the sampling of two channels at the Nyquist rate, sampling and data throughput rates can also be demanding.

In this paper, a new FMCW radar architecture that implements a novel technique of synchronising nodes in a multistatic system, known as over-the-air deramping (OTAD), is proposed and developed. The architecture includes a cooperative FMCW transmitter and many passive nodes to form an adjustable coverage area. By using an FMCWbased approach, OTAD overcomes PBR limitations to make significant improvements in range-resolution and sampling rate requirements. Furthermore, with a dual-frequency design, the limiting effect of direct signal interference is avoided.

The following section presents a detailed explanation of the OTAD technique, including a mathematical description and predictive performance equations. Following this is a description of Soprano, the first radar system developed to test the OTAD technique. Then the results of some measurements of stationary and moving targets taken using two Soprano radar 


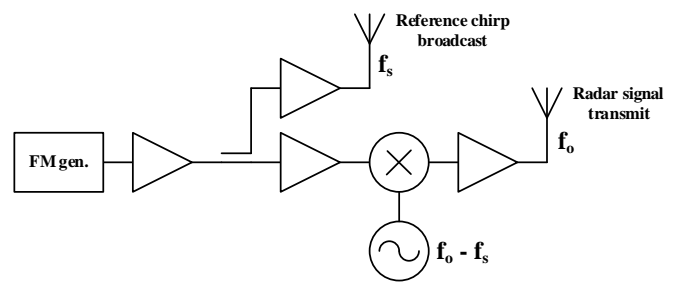

(a)

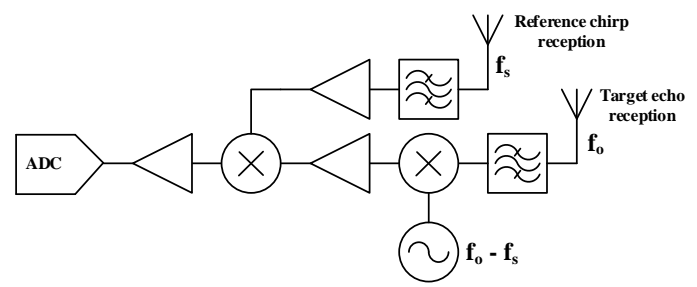

(b)

Fig. 1. Simplified block diagram of an FMCW over-the-air deramping (a) Master node and (b) Slave node architecture.

nodes, forming a bistatic radar system, are shown. Finally, the results from the measurements are discussed, and conclusions are drawn.

\section{OVER-THE-AIR DERAMPING}

An OTAD radar system consists of distributed sensors including a master node and $N$ slave nodes. Fig. 1 shows a simplified block diagram of the construction of a master and a slave node. A master node consists of a full FMCW transmitter with a key modification to its architecture; a portion of the FMCW radar signal, or chirp signal, is tapped off at an IF frequency, $f_{\mathrm{s}}$, as in a super-heterodyne FMCW radar system and fed into an antenna, such as a horizontally omnidirectional antenna, for broadcast as a reference chirp signal. The chirp signal is simultaneously translated to an operating frequency, $f_{\mathrm{o}}$, for transmission towards a scene to be measured. Hence, the reference chirp signal and the radar signal operate at different frequencies.

On reception, a slave node directs one antenna to face the reference chirp broadcast (master) node and another towards the measurement scene. The received reference chirp (overthe-air deramp) signal is filtered and amplified to a signal level sufficient to drive a deramping mixer. In parallel, the target echo signal at $f_{\mathrm{o}}$ is filtered, amplified and translated to an IF equal to $f_{\mathrm{s}}$ before entering the deramp mixer. The output of the deramping process is a beat signal whose frequency is proportional to the bistatic path to the target less the line-ofsight distance between the master node broadcast antenna and the slave node reference chirp-facing antenna.

By operating the over-the-air deramp channel and the target echo channel at different frequencies, OTAD sensors can be arranged in any configuration without direct signal interference issues. An example configuration is shown in Fig. 2, which depicts the broadcast and reception of the over-the-air deramp signal. The slave nodes employ directional antennas

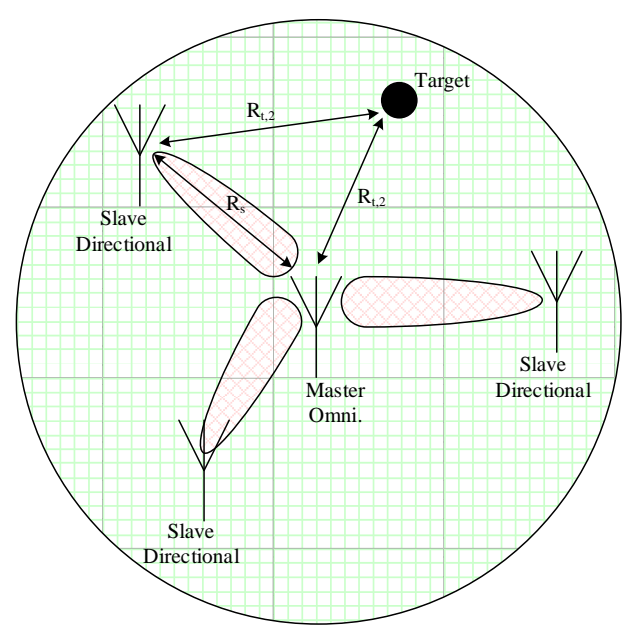

Fig. 2. Configuration of a multistatic over-the-air deramping system showing coverage regions of reference chirp broadcast. Master node coverage area is omnidirectional (green region) and slave nodes point directive antennas (red regions) towards the master node.

on reception in order to reduce the susceptibility to multipath. This arrangement allows many slave nodes to be deployed and distributed to form coverage over areas at a modest additional hardware cost.

\section{A. Deramp Signal Analysis}

As described in the previous section, the master transmitter continuously transmits a chirp waveform, simultaneously on two channels, of the form [1]:

$$
x_{\mathrm{o}}(t)=a_{\mathrm{o}} \cos 2 \pi\left[f_{\mathrm{o}} t+(1 / 2) \alpha t^{2}\right]
$$

where $a_{\mathrm{o}}$ is the signal amplitude, $f_{\mathrm{o}}$ is the chirp start frequency and $\alpha$ is the chirp rate (the ratio of the chirp bandwidth and the chirp period, $B / T)$. The transmitted waveform reaches the two receiver elements following some propagation delay, $\tau$

$$
\begin{aligned}
& x_{\mathrm{s}}(t)=a_{\mathrm{s}} \cos 2 \pi\left[f_{\mathrm{o}}\left(t-\tau_{\mathrm{s}}\right)+(1 / 2) \alpha\left(t-\tau_{\mathrm{s}}\right)^{2}\right] \\
& x_{\mathrm{t}}(t)=a_{\mathrm{t}} \cos 2 \pi\left[f_{\mathrm{o}}\left(t-\tau_{\mathrm{t}}\right)+(1 / 2) \alpha\left(t-\tau_{\mathrm{t}}\right)^{2}\right]
\end{aligned}
$$

where $x_{\mathrm{s}}$ is the over-the-air deramp signal, and $x_{\mathrm{t}}$ is the target echo signal. To simplify the analysis, it has been assumed that the reference deramp and echo signals are at the same operating frequency, i.e. ideal downconversion of the echo signal in the receiver. Conventional FMCW radar would simply mix a portion of the transmitted signal, $x_{\mathrm{o}}$, and the signal associated with the reflection from the target, $x_{t}$, to give a deramp signal of the following form (after low-pass filtering):

$$
\begin{aligned}
& y_{\mathrm{d}}(t)=x_{\mathrm{o}}(t) \cdot x_{\mathrm{t}}(t) \\
& y_{\mathrm{d}}(t)=d_{\mathrm{o}} \cos 2 \pi\left[f_{\mathrm{o}} \tau_{\mathrm{t}}+\alpha t \tau_{\mathrm{t}}-(1 / 2) \alpha \tau_{\mathrm{t}}^{2}\right]
\end{aligned}
$$

The second term, known as the deramp frequency $f_{\mathrm{d}}$, can then be related to range by the following relationship:

$$
f_{\mathrm{d}}=\alpha \tau_{\mathrm{t}}=\frac{2 B R_{\mathrm{t}}}{c T}
$$


where $\tau_{\mathrm{t}}=2 R_{\mathrm{t}} / \mathrm{c}$ is the two-way propagation delay to the target.

Over-the-air deramping involves mixing the target signal and the over-the-air deramp signal as follows:

$$
\begin{aligned}
y_{\mathrm{d}}(t) & =x_{\mathrm{s}}(t) \cdot x_{\mathrm{t}}(t) \\
y_{\mathrm{d}}(t) & =g_{\mathrm{o}} \cos 2 \pi\left[f_{\mathrm{o}}\left(\tau_{\mathrm{t}}-\tau_{\mathrm{s}}\right)+\alpha t\left(\tau_{\mathrm{t}}-\tau_{\mathrm{s}}\right)\right. \\
& \left.-(1 / 2) \alpha\left(\tau_{\mathrm{t}}^{2}-\tau_{\mathrm{s}}^{2}\right)\right]
\end{aligned}
$$

Hence, the deramp frequency becomes a function of the lineof-sight propagation delay between the master and slave nodes, and the bistatic propagation delay to the target. For a bistatic scenario, $R_{\mathrm{t}}$ is expanded to $R_{\mathrm{t}, 1}+R_{\mathrm{t}, 2}$ modifying the deramp frequency to

$$
\begin{aligned}
& f_{\mathrm{d}}=\alpha\left(\tau_{\mathrm{t}}-\tau_{\mathrm{s}}\right) \\
& f_{\mathrm{d}}=\frac{B\left(R_{\mathrm{t}, 1}+R_{\mathrm{t}, 2}\right)}{c T}-\frac{B R_{\mathrm{s}}}{c T}
\end{aligned}
$$

The expression of the OTAD deramp signal in (6) can be expanded to include relative motion, $v$, of a target in the field of view, i.e. $\tau_{\mathrm{t}}=2 r_{\mathrm{t}} / c+2 v t / c$, thus

$$
\begin{aligned}
y_{\mathrm{d}}(t)=g_{\mathrm{o}} \cos 2 \pi\left[\frac { 2 f _ { \mathrm { o } } } { c } \left(r_{\mathrm{t}}\right.\right. & \left.-r_{\mathrm{s}}\right)+\frac{2 \alpha}{c}\left(r_{\mathrm{t}}-r_{\mathrm{s}}\right) t \\
+ & \left.\frac{2 f_{\mathrm{o}} v t}{c}+\frac{2 \alpha v t^{2}}{c}\right]
\end{aligned}
$$

where it has been assumed that the two radar nodes have zero relative motion, and the third term of (6) can be ignored assuming that the propagation time delay is much less than the chirp period, i.e. $\tau_{\mathrm{t}} \ll T$; $\tau_{\mathrm{s}} \ll T$. The third term of (8) can be estimated from measurements using triangular modulation of the chirp waveform or two-dimensional Fourier analysis [14]. The fourth term expresses delay-Doppler cross-coupling [1]. Expanding $R_{\mathrm{t}}$, the moving target deramp frequency is

$$
f_{\mathrm{d}}=\frac{B\left(R_{\mathrm{t}, 1}+R_{\mathrm{t}, 2}\right)}{c T}-\frac{B R_{\mathrm{s}}}{c T}+\frac{2 f_{\mathrm{o}} v}{c}
$$

\section{B. OTAD Radar Performance Equations}

The radar performance can be analysed with familiar bistatic radar approaches. The range resolution, $\Delta R$, of the chirp signal employed by an OTAD system, and the maximum unambiguous range, $R_{\max }$, are given by the following expressions [15]:

$$
\begin{aligned}
\Delta R & =\frac{c}{2 B} \\
R_{\max } & =\frac{c f_{\mathrm{n}} T}{4 B}, \quad R_{\mathrm{t}}>R_{\mathrm{s}}
\end{aligned}
$$

where $B$ is the chirp bandwidth and $f_{\mathrm{n}}$ is the baseband sampling rate.

Considering thermal noise of an FMCW receiver with a bandwidth of $1 / T$ (following deramping) and a noise factor, $F$, the thermal-noise limited SNR performance of a bistatic OTAD receiver is:

$$
S N R=\frac{P_{\mathrm{t}} G_{\mathrm{t}} G_{\mathrm{r}} \lambda_{\mathrm{o}}^{2} \sigma T L_{\mathrm{p}}}{(4 \pi)^{3} R_{\mathrm{t}, 1}^{2} R_{\mathrm{t}, 2}^{2} k T_{\mathrm{o}} F}
$$

where $P_{\mathrm{t}}$ is the average transmit power (equivalent to the peak transmit power in FMCW systems), $G_{\mathrm{t}}$ and $G_{\mathrm{r}}$ are the antenna gains of the transmitter node and the receiver node respectively, $\lambda_{\mathrm{o}}$ is the radar signal wavelength, $\sigma$ is the target radar cross section (RCS), $L_{\mathrm{p}}$ accounts for losses associated with the propagation medium, $R_{\mathrm{t}, 1}+R_{\mathrm{t}, 2}\left(=R_{\mathrm{t}}\right)$ is the bistatic path distance, $k$ is the Boltzmann constant and $T_{\mathrm{o}}$ is the ambient temperature. However, this equation does not consider the noise introduced by the over-the-air deramp signal channel, which operates at $f_{\mathrm{s}}$.

\section{OTAD Noise Analysis}

The use of an over-the-air deramp signal has some effect on the performance of the system relative to the use of a conventional wired deramp signal. This effect can be demonstrated with a noise analysis of the deramping process. Fig. 3 depicts a simplified OTAD FMCW radar front-end, comprising an echo path LNA of gain $G_{1}$, a deramp signal path LNA of gain $G_{2}$ and a mixer that is used to perform the deramp process. The noise factors of the two LNAs, $F$, are assumed to be similar. The mixer input noise power spectral density in the echo path is thus

$$
N_{1}=k T G_{1} F \quad[\mathrm{~W} / \mathrm{Hz}]
$$

The required LNA gain, $G_{2}$, in the deramp signal path is equal to the ratio of the required mixer local oscillator (LO) power, $P_{\mathrm{LO}}$, and the input deramp signal level, $P_{\text {chirp }} G_{\text {ant }} / L$, where $P_{\text {chirp }}$ is the effective radiated power (EIRP) of the chirp (deramp) signal, $L$ is the free-space path loss and $G_{\text {ant }}$ is the gain of the reference chirp-facing antenna. Hence the mixer input noise density in the deramp path is

$$
\begin{aligned}
N_{2} & =k T G_{2} F \\
& =k T F \frac{P_{\mathrm{LO}} L}{P_{\text {chirp }} G_{\text {ant }}}
\end{aligned}
$$

where the free-space path loss factor at $f_{\mathrm{s}}$ is

$$
L=\left(\frac{4 \pi R_{\mathrm{s}}}{\lambda_{\mathrm{s}}}\right)^{2}
$$

If it is assumed that the mixer is driven close to saturation on the LO port, then the additive noise contributed in this path is one-half of the value of (14) [16]. The action of the mixer is to overlay the two noise sources $N_{1}$ and $N_{2}$ so that the total noise density at the output (assuming high amplifier gains and thus minimal second-stage noise effects) is

$$
\begin{aligned}
N_{\text {out }} & =N_{1}+N_{2} / 2 \\
& =k T F\left(G_{1}+\frac{P_{\mathrm{LO}} L}{2 P_{\text {chirp }} G_{\text {ant }}}\right)
\end{aligned}
$$

In the absence of noise on the deramp signal (LO) port, the mixer output noise would simply be $N_{1}$ and thus the degradation in noise is given by

$$
\frac{N_{\text {out }}}{N_{1}}=1+\frac{P_{\mathrm{LO}} L}{2 P_{\text {chirp }} G_{1} G_{\text {ant }}}
$$

Taking the newly developed Soprano system (discussed later) as an example, with an echo path LNA gain of $30 \mathrm{~dB}$, a required mixer LO level of $+4 \mathrm{dBm}$, a chirp-facing antenna gain of $12 \mathrm{dBi}$ and a centre frequency of $2.45 \mathrm{GHz}$ and 


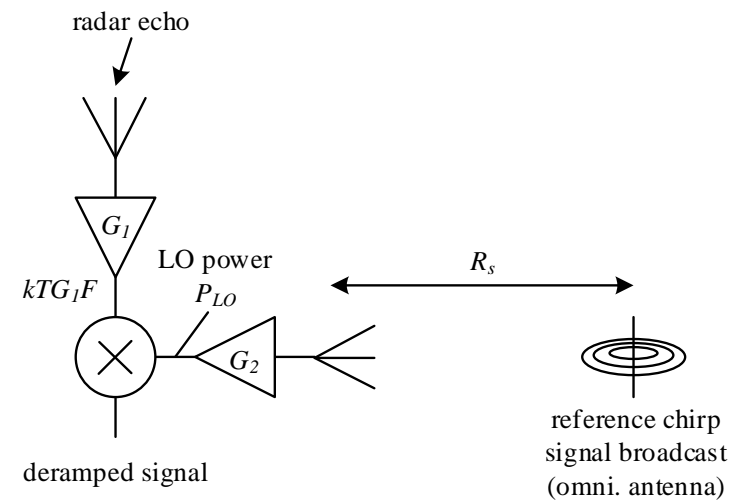

Fig. 3. Front-end arrangement of an FMCW radar employing the OTAD technique showing noise at the deramp mixer inputs.

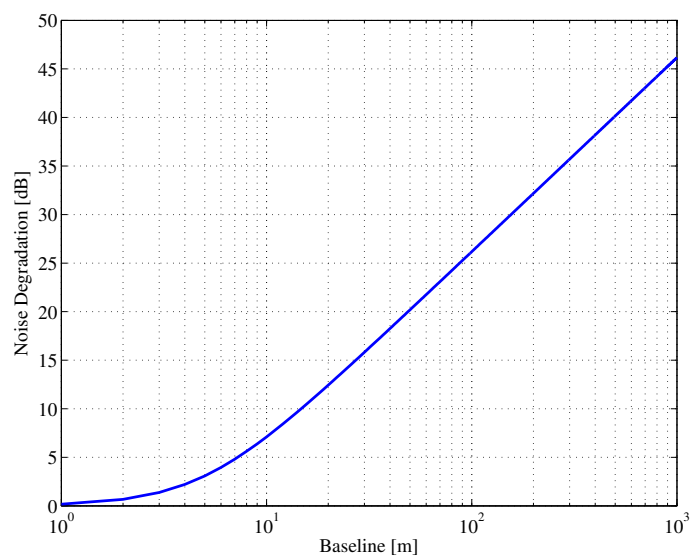

Fig. 4. Noise degradation versus range to chirp broadcast antenna (baseline); $2.45 \mathrm{GHz}, G_{1}=30 \mathrm{~dB}, P_{L O}=4 \mathrm{dBm}, G_{a n t}=12 \mathrm{dBi}$.

EIRP of $+13 \mathrm{dBm}$, (17) indicates a noise (and hence SNR) degradation of $7 \mathrm{~dB}$ at $10 \mathrm{~m}$ and $26 \mathrm{~dB}$ at $100 \mathrm{~m}$. Fig. 4 shows the variation of noise level degradation with range subject to these parameters. Clearly upon inspection of (17) it is advantageous to maximise the echo path LNA and chirp-facing antenna gains.

\section{Mitigating The Effect of Master-Slave LO Drift}

OTAD, in the form used by the system described in this paper, uses separate downconversion LOs on the master node and the slave node to translate the chirp signal from the operating frequency on reception. This means that it is not a fully coherent system. However, the effect of drift between operating frequency of the nodes forming a bistatic pair can be mitigated when a high-SNR, stationary target exists within the measurement scene.

The LO signal can be assumed to be driving the LO port of the downconversion mixer in the slave receiver close to saturation, hence its time varying behaviour, ignoring phase noise, can be modelled as

$$
V(t)=\cos \left[\left(\omega_{\mathrm{LO}}+\Delta \omega_{\mathrm{LO}}(t)\right) t\right]
$$

where $\omega_{\mathrm{LO}}$ is the angular frequency of the LO on the master node. The second term implies a time-varying frequency error

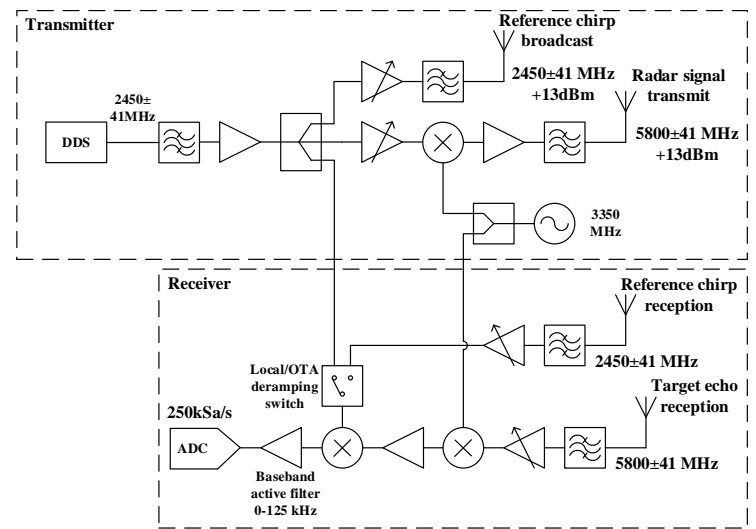

Fig. 5. Simplified system block diagram of Soprano FMCW OTAD radar.

relative to LO driving the master node. The presence of this frequency error in the downconversion mixer means that it is also captured in the deramp signal following a downconversion process prior to deramping. This modifies (4) to

$$
\begin{gathered}
y_{\mathrm{d}}(t)=d_{\mathrm{o}} \cos 2 \pi\left[f_{\mathrm{o}} \tau_{\mathrm{t}}+\alpha \tau_{\mathrm{t}} t-(1 / 2) \alpha \tau_{\mathrm{t}}^{2}\right. \\
\left.+\Delta f_{\mathrm{LO}}(t) t\right]
\end{gathered}
$$

Hence, the deramp signal of a stationary target provides the opportunity to estimate this error term through adequate timefrequency analysis. Following estimation, a compensation to the entire range profile can be applied as the errors are independent of range.

\section{Soprano System DeVElopment}

In order to fully analyse the OTAD technique, a radar that utilises the OTAD architecture with a monostatic FMCW radar as an optional mode was developed. This allows for simultaneous monostatic and multistatic measurements and, hence, direct comparison between the two. The radar system is known as Soprano and to date two radar nodes of the same design have been constructed.

Soprano was designed to be easily scalable so that more radar nodes can be constructed for further experiments with only modest additional technical input. The entire design is PCB-based with commercial off-the-shelf surface mount components. A simplified block diagram of the system is shown in Fig. 5 and a summary of its specifications is shown in Table I. Fig. 6 shows an image of the Soprano PCB.

As per the OTAD architecture, Soprano has two transmitter and two receiver channels. The reference chirp channel operating frequency is $2.45 \mathrm{GHz}$, and the target echo channel operating frequency is $5.8 \mathrm{GHz}$. The design is based on the use of a DDS chip. The DDS is clocked by an integrated VCO and PLL chip tuned to $3.35 \mathrm{GHz}$. This allows for the generation of linear frequency modulated continuous wave signal with a $2.45 \mathrm{GHz}$ centre frequency by using the superNyquist technique [17], which is well suited to multistatic FMCW radar [18]. In this case, the image of a $900 \mathrm{MHz}$ chirp signal is selected from the first Nyquist zone using a bandpass filter. This signal is split for local deramping 
(forming a monostatic node), for transmission as the overthe-air deramp signal (forming the bistatic channel), and for upconversion to the target echo channel operating frequency. Translation to $5.8 \mathrm{GHz}$ is achieved by mixing the deramp signal with a portion of the $3.35 \mathrm{GHz}$ DDS clock signal. Each channel has the potential for transmission of up to $200 \mathrm{MHz}$ bandwidth, though in the experiments described in this paper, the bandwidth was limited to $83.5 \mathrm{MHz}$ to adhere to UK Ofcom regulations.

On reception, an RF switch is used to select the mode of the radar node (master or slave). In master mode, the RF switch selects the local deramp signal path to form a monostatic radar. In slave mode, the RF switch selects the over-the-air deramp signal path. In this path, there is a significant amount of available gain and channel selection filters. This is so that the gain of this channel can be adjusted depending on the distance between the master broadcast antenna and the slave chirp-facing antenna. The required gain can be estimated by (using the same nomenclature as Section II-C):

$$
G_{2}=\frac{P_{\mathrm{LO}} L}{P_{\text {chirp }} G_{\text {ant }}}
$$

As an illustration, a master node with a $+13 \mathrm{dBm}$ EIRP and a slave node with a antenna gain of $12 \mathrm{dBi}$, and a deramp mixer with $\mathrm{LO}$ drive level of $+4 \mathrm{dBm}$, the required gain in the over-the-air deramp signal path is some $39 \mathrm{~dB}$ with a baseline separation of $10 \mathrm{~m}$.

Following deramping, the signal is conditioned by a baseband active filter, which performs frequency-gain control to make maximum use of the ADC dynamic range [1]. The ADC used to record the measurements in this work was a National Instruments USB-6341 with 16-bit resolution and operating with a $250 \mathrm{kSa} / \mathrm{s}$ sampling rate.

Each radar node is programmed individually using an external microcontroller to set the radar signal parameters and the transmit power and receiver gain.

In monostatic mode, the radar node is fully coherent with all of the LOs and ADC clock derived from the same $10 \mathrm{MHz}$ reference clock. In multistatic mode, the downconversion LO is not coherent with the transmitter. Hence, a slave node forms an incoherent bistatic pair. However, using the method described in Section II-D, it will be shown in measurement that it is possible to compensate for this issue in processing with the presence of a high-SNR stationary target within the measurement scene.

Our measurements with this system primarily focus on proving its effectiveness as a short-range surveillance system, hence humans were chosen as targets during the experiments. The thermal noise limited SNR of an echo from a human target can be estimated using (12) and typical Soprano radar parameters of a chirp period of $1 \mathrm{~ms}$, antenna gain of $12 \mathrm{dBi}$ on each antenna and a baseline separation of $10 \mathrm{~m}$. Such a baseline degrades the noise figure by some $7 \mathrm{~dB}$ using the same reference chirp parameters as in Section II-C. The noise figure of the system varies with frequency as a result of the frequency gain control, with a typical value of $2.5 \mathrm{~dB}$ in monostatic mode and $9.5 \mathrm{~dB}$ in OTAD mode. Using a human RCS of $1 \mathrm{~m}^{2}$ [15],

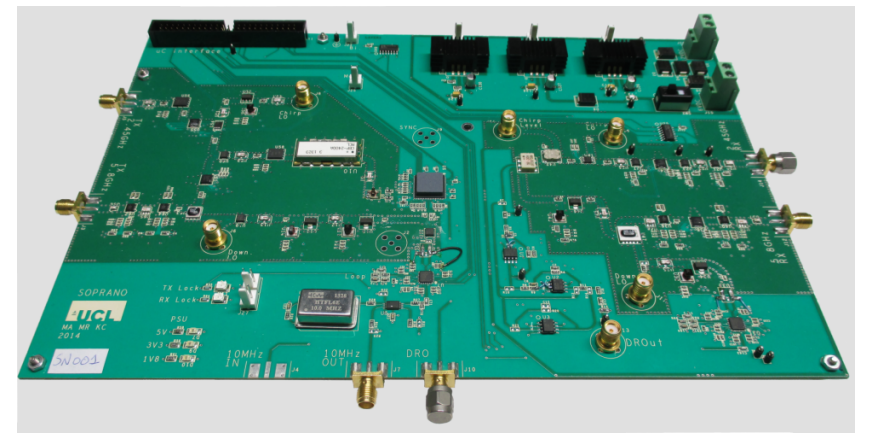

Fig. 6. Soprano FMCW OTAD radar PCB.

TABLE I

SOPRANO FMCW OTAD RADAR SPECIFICATION.

\begin{tabular}{lc}
\hline \hline Centre Frequency & $5.8 \mathrm{GHz}$ \\
\hline Transmit Power & $+13 \mathrm{dBm}$ \\
\hline Waveform Bandwidth & $83.5 \mathrm{MHz}$ \\
\hline Base-bandwidth & $125 \mathrm{kHz}$ \\
\hline Noise Figure & $2.5 \mathrm{~dB}$ \\
\hline Phase Noise & \\
@ $1 \mathrm{kHz}$ & $-83 \mathrm{dBm} / \mathrm{Hz}$ \\
@ $100 \mathrm{kHz}$ & $-85 \mathrm{dBm} / \mathrm{Hz}$ \\
@ $1 \mathrm{MHz}$ & $-125 \mathrm{dBm} / \mathrm{Hz}$ \\
\hline
\end{tabular}

Fig. 7 shows that a human target is theoretically measurable and detectable at bistatic ranges beyond $200 \mathrm{~m}$.

\section{Field Measurements}

In order to demonstrate the OTAD technique, several field experiments were conducted in Wimbledon, UK, January 2015. The objectives of the measurements were to prove that OTAD works for measurements of stationary targets and moving humans, and to compare OTAD measurements to simultaneously recorded conventional monostatic measurements.

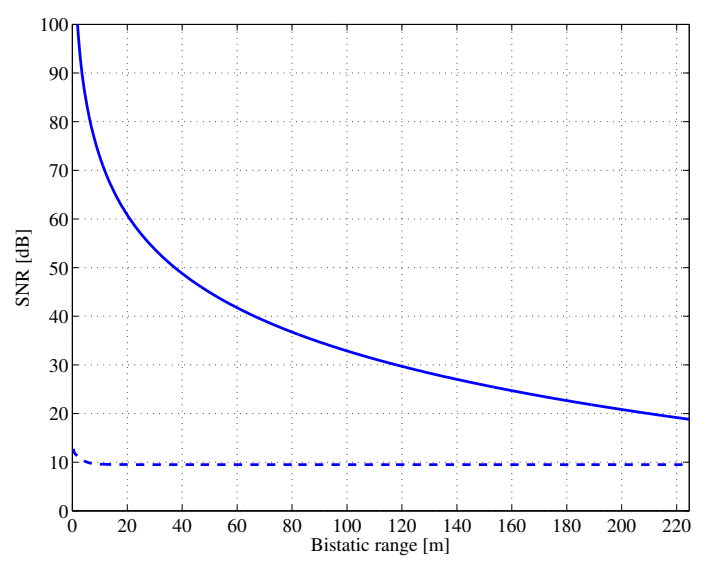

Fig. 7. Predicted SNR versus range assuming a $1 \mathrm{~ms}$ chirp duration and a human target with a RCS of $1 \mathrm{~m}^{2}$ as measured by a bistatic node in Soprano radar system with a baseline of $10 \mathrm{~m}$. The Soprano receiver noise figure has also been included (dashed). 


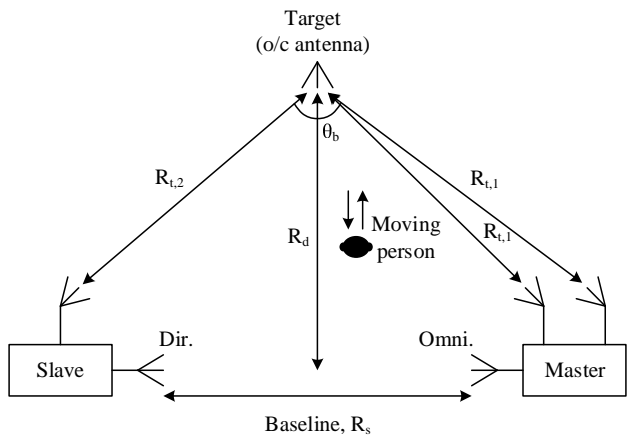

(a)

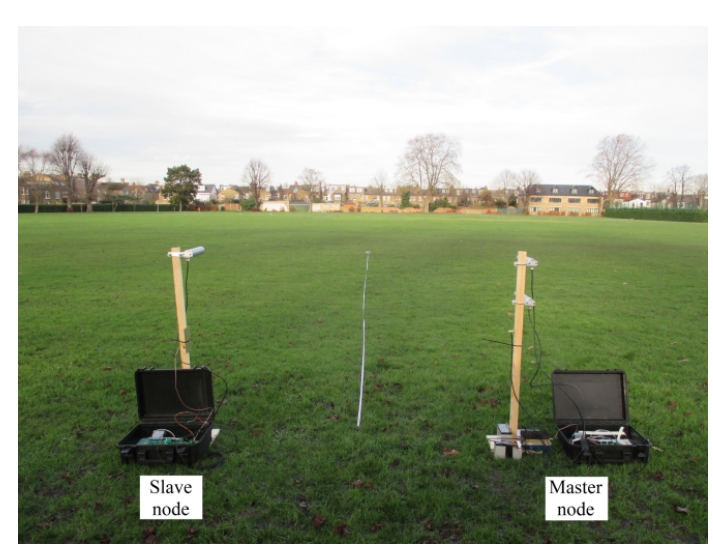

(b)

Fig. 8. Experiment setup (a) diagram (b) photograph.

\section{A. Experiment Setup}

Two Soprano radar nodes were used in the experiments; one was configured as a master node and the other as a slave node. The two nodes were separated to give a baseline separation $R_{\mathrm{s}}$. Fig. 8 shows a diagram of the experiment setup and a photograph of the location. The target facing antennas were pointed towards a field, which was largely free of clutter. The field was fringed by residential housing at a range of some $160 \mathrm{~m}$.

Throughout the measurements, the transmit power was kept at its maximum level $(+13 \mathrm{dBm})$. The chirp period was set to $1 \mathrm{~ms}$ and bandwidth to $83.5 \mathrm{MHz}$, and a sawtooth modulation scheme was used (i.e. up chirps only). Such waveform parameters give a nominal range resolution of $1.8 \mathrm{~m}$. The over-theair deramp signal was broadcast using a c. $2 \mathrm{dBi}$ horizontally omnidirectional antenna and the chirp-facing antenna on the slave node had a gain of c. $12 \mathrm{dBi}$. Each of the $5.8 \mathrm{GHz}$ radar channel antennas were $30^{\circ}$ yagi antennas with a gain of $12 \mathrm{dBi}$. The antennas were mounted $1.5 \mathrm{~m}$ above the ground.

\section{B. Stationary Target Measurements}

The stationary target used was a panel antenna with an open circuit on its feed. The antenna has a horizontal beamwidth of $30^{\circ}$ and a gain of $12 \mathrm{dBi}$. It was mounted at a height of $1.5 \mathrm{~m}$ above the ground and placed at three locations. The three locations were $R_{\mathrm{d}}=20,30$ and $50 \mathrm{~m}$ from the centre of the baseline as shown in the diagram of the geometry in Fig. 8a. The target was measured with two baseline separations,

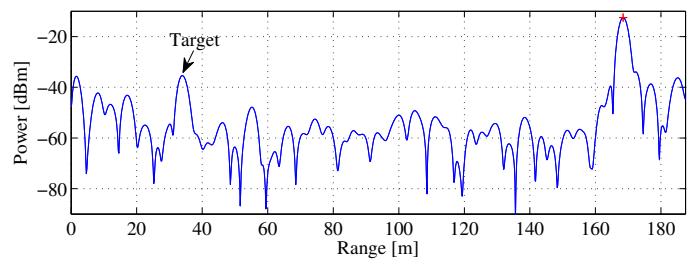

(a)

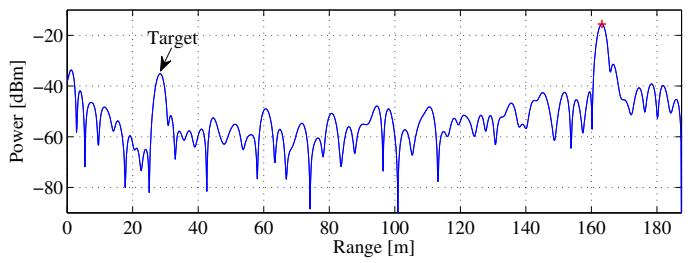

(b)

Fig. 9. (a) Monostatic measurement of stationary target at $30 \mathrm{~m}$ range at master node and (b) bistatic measurement at slave node with a $5 \mathrm{~m}$ baseline The location of the buildings fringing the field in which these measurements were taken are marked with a red cross.

$R_{\mathrm{s}}=5$ and $10 \mathrm{~m}$, in order verify the theory presented in Section II-A. These geometries created various bistatic angles between $5.7^{\circ}$ and $28.1^{\circ}$.

Range processing was carried out by splitting the recorded deramp signal into individual chirps and applying a Hanning window to each individual chirp. A zero-padded FFT with a padding factor of 10 was then performed on each individual chirp and the value of associated frequency bins were converted to range using (5) in order to directly compare the monostatic and OTAD bistatic measurements.

The stationary target was visible from both radar nodes. Fig. 9 shows the range profile of single chirp capture with the stationary target at $R_{\mathrm{d}}=30 \mathrm{~m}$ with a $5 \mathrm{~m}$ baseline, which forms a bistatic angle of $9.2^{\circ}$. Table II summarises the results from all of the geometries averaged over a $10 \mathrm{~s}$ capture. The nominal range resolution is $1.8 \mathrm{~m}$, which implies a accuracy of $\pm 0.9 \mathrm{~m}$. However, following Hanning windowing the measurement accuracy is $\pm 1.3 \mathrm{~m}$. The typical measurement standard deviation is between $0.1-0.3 \mathrm{~m}$, depending on the range to the target (increasing with range).

The results show a consistent offset on all the monostatic measurements of around $4 \mathrm{~m}$. This is due to the cabling running to and from the transmit and receive antennas on the target echo channel. The measurements of the target at $50 \mathrm{~m}$ are roughly $1-2 \mathrm{~m}$ beyond expected, considering cable lengths. This is thought to be a result of human error in the manual placement of the target at longer ranges. Comparing the $5 \mathrm{~m}$ and the $10 \mathrm{~m}$ baseline measurements, there is roughly a $5.5 \mathrm{~m}$ and $8 \mathrm{~m}$ offset respectively to the slave node measurements relative to the master node measurements. Removing the factor of two the range processing (using (5)) applies, the offsets become $11 \mathrm{~m}$ and $16 \mathrm{~m}$. Accounting for the extra offsets due to the cabling running to and from the transmit and receive antennas on the OTAD channel (c. $2 \mathrm{~m}$ more than the monostatic cabling), this result shows a good agreement with (7). 
TABLE II

COMPARISON OF EXPECTED (EXP.), MEAN OF THE MONOSTATIC (MONO.) AND MEAN OF THE BISTATIC OTAD MEASUREMENTS OF A STATIONARY TARGET WITH 5 M AND $10 \mathrm{M}$ BASELINES AT VARIOUS RANGES.

\begin{tabular}{lcccccc}
\hline \hline Range $[\mathrm{m}]$ & \multicolumn{3}{c}{ 5m Baseline } & \multicolumn{3}{c}{ 10m Baseline } \\
\hline $\mathrm{R}_{\mathrm{d}}$ & Exp. & Mono. & OTAD & Exp. & Mono. & OTAD \\
\hline 20 & 20.2 & 24.0 & 18.6 & 20.6 & 24.2 & 17.0 \\
\hline 30 & 30.1 & 34.2 & 28.7 & 30.4 & 35.0 & 27.0 \\
\hline 50 & 50.1 & 55.3 & 49.1 & 50.3 & 56.3 & 48.0 \\
\hline
\end{tabular}

\section{Moving Target Measurements}

Moving target measurements were carried out with a walking person. In the dataset discussed in this paper, the person walked away from the radar nodes at the centre of the baseline, and then turn around at c. $50 \mathrm{~m}$ from the baseline where the stationary target was positioned, and walked back to the radar nodes. The baseline was $5 \mathrm{~m}$.

It was discussed in Section II-D that the use of separate LOs on the master node and the slave node will produce errors in the deramp signal measurement. The errors manifest themselves as time-varying frequency offset. In this dataset, the effect that this issue has on the data is apparent when performing Doppler and moving target indication (MTI) processing. In Section II-D, it was proposed that with the measurement of high-SNR stationary target, these errors could be corrected for in the entire measurement scene as they are not range-dependent. The buildings towards the edge of the field provided a higher SNR target (indicated with a red cross in Fig. 9) and hence their response was used as a synchronising target.

In the results shown in this paper, a simple, first-order correction was used; it was assumed that the frequency error would stay constant during the period of at least two chirps. Hence, the frequency error, $\Delta \hat{\omega}_{\mathrm{LO}}$, could be estimated from

$$
\Delta \hat{\omega}_{\mathrm{LO}}=\frac{\hat{\phi}_{0, \mathrm{n}+1}-\hat{\phi}_{0, \mathrm{n}}}{T}
$$

where $n$ is the chirp number, $\hat{\phi}_{0}$ is an estimate of the phase synchronising target. Using this assumption, all range bins are phase wrapped by $-\hat{\phi}_{0}$ in order to correct the data.

Following correction, the data was passed through a moving target indication (MTI) filter based on an extension to the moving-average high-pass filter described by Stove [1]. The filter used was a 100 order high-pass Chebyshev FIR filter with a cut-off frequency of $10 \mathrm{~Hz}$ (equating to a speed of $0.25 \mathrm{~m} / \mathrm{s}$ ).

Fig. 10 shows range-time plots of the MTI filtered data from a measurement of a person moving within the field-of-view. Fig. 10b shows the negative effect of master-slave LO drift on MTI performance with the stationary target positioned at $50 \mathrm{~m}$ still clearly visible. Applying the aforementioned correction technique improves the MTI performance dramatically, as shown in Fig. 10c. Following MTI filtering, the presence of a moving target is clear in both the monostatic and the OTAD bistatic measurements. The SNR approaches $30 \mathrm{~dB}$ at $50 \mathrm{~m}$ range for both measurements, with the OTAD bistatic measurements some $2 \mathrm{~dB}$ lower than the monostatic measurements, which is close to the noise degradation of $3 \mathrm{~dB}$ predicted by (17). The stationary target has been suppressed by more than $30 \mathrm{~dB}$ in both cases. Hence, moving targets and stationary targets could be distinguished with simple detectors such as a order-statistic constant false alarm rate detector [19].

The MTI filtered OTAD bistatic image shows the appearance of a ghost target $3.5 \mathrm{~m}$ up-range from the main target. This is thought to be a result of multipath in the over-the-air deramp channel. During the measurement, there was a stationary clutter located a few metres behind the master radar node, such as chairs and equipment. In this case, the multipath has negligible effect on the results of the measurement, however it does highlight the need to be cautious in more complex environments and to look to employ methods of mitigating the effect of multipath. Additionally, following application of the correction procedure, a response appears at c. $1 \mathrm{~m}$ range. This is a spurious response generated locally in the receiver.

\section{CONClusions AND Future Work}

In this paper, a new FMCW radar architecture called overthe-air deramping (OTAD) has been introduced that facilitates multistatic systems. The architecture, which separates a synchronisation chirp signal and the radar signal in frequency, allows for any node configuration without suffering from direct signal interference normally associated with passive bistatic radar nodes. Furthermore, by using deramping, good range resolution is achievable with only modest sampling rate and data throughput requirements.

A simple mathematical analysis shows that the deramp signal frequency is a function of the bistatic range and the baseline separation. The bistatic radar equation is used to examine the performance in terms of signal-to-noise ratio. It was found through analysis of the deramp mixing process that the use of an over-the-air deramp signal channel adds noise to system relative to a conventional monostatic deramping system, at a level that is baseline separation dependent.

A new $5.8 \mathrm{GHz}$ FMCW OTAD system, called Soprano, has been introduced in this paper. Soprano has been used to demonstrate and test the OTAD technique with real measurements of humans. The system is capable carrying out monostatic and bistatic OTAD measurements simultaneously. The design provides a theoretical SNR of more than $30 \mathrm{~dB}$ at $100 \mathrm{~m}$ bistatic range with a $10 \mathrm{~m}$ baseline. Stationary target measurements agree well with the aforementioned mathematical analysis. A correction using the measurement of a highSNR stationary target was applied to the bistatic OTAD data in order to mitigate the effect of drift between the separate master and slave LOs. Following this correction, the performance of an MTI filter is dramatically improved, and the resultant rangetime image shows good SNR responses (consistently $>20 \mathrm{~dB}$ ) to a moving person.

To date, the OTAD technique has been tested with a bistatic geometry, limited by the number of available Soprano nodes. This work will be taken forward with the construction of more Soprano nodes to form a multistatic system. Our hypothesis is that the increased information content of the multistatic data recorded from a well-designed deployment of the hardware 


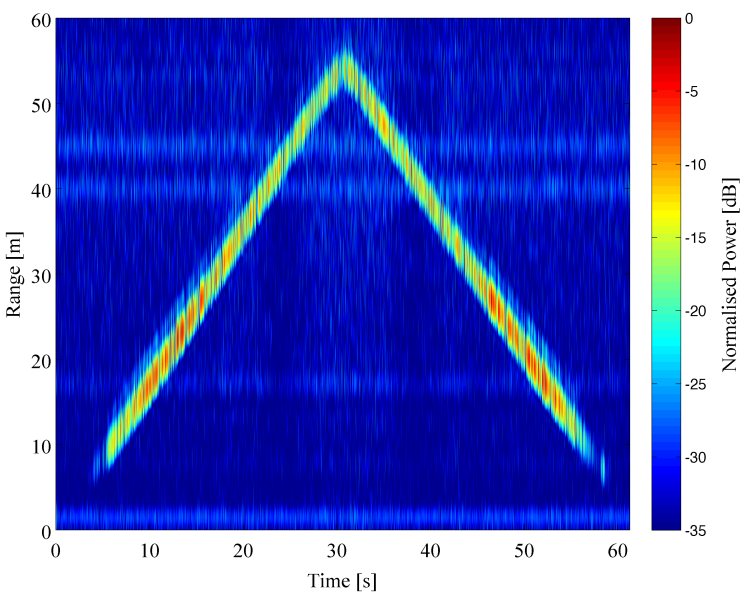

(a)

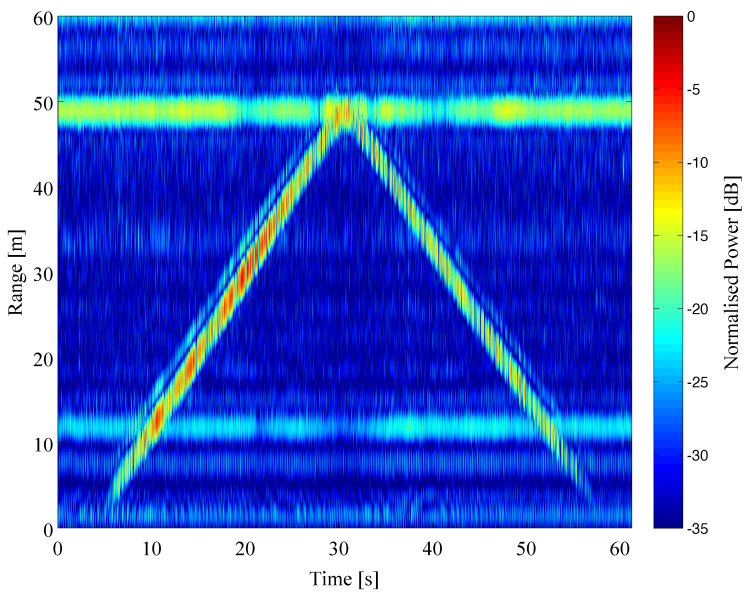

(b)

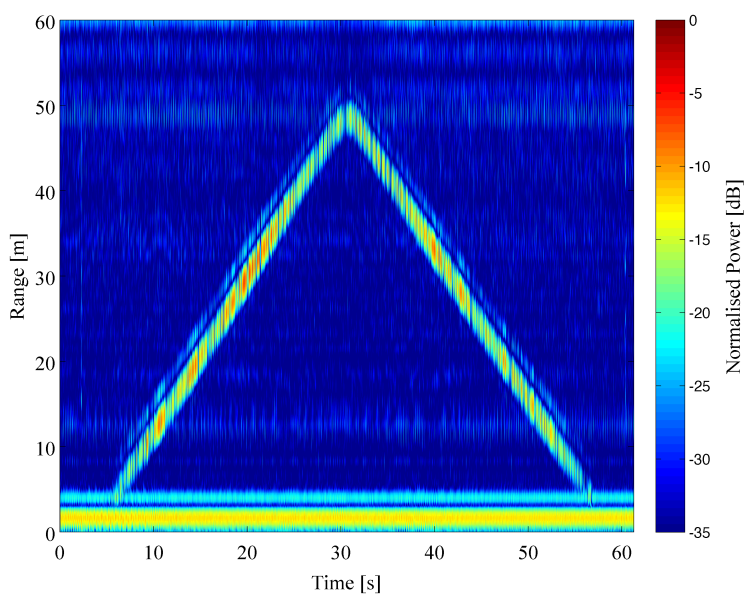

(c)

Fig. 10. Range-time images of person walking away from radar and towards radar. (a) Monostatic measurement at master node following MTI filtering with a $10 \mathrm{~Hz}$ cut-off. (b) Bistatic OTAD measurement at slave node following MTI filtering prior to correcting for master-slave node LO incoherence and (c) bistatic OTAD measurement following correction of of master-slave node LO incoherence. will increase the effectiveness of classification techniques. With this capability, the OTAD technique becomes a compelling solution for many applications including surveillance and security.

\section{ACKNOWLEDGMENT}

The authors would like to thank the UCL Institute of Making for funding the hardware component of this project through the EPSRC Bridging the Gaps grant. Matthew Ash would like to further thank the EPSRC for their support of his research through grant ref. EP/K00767X/1.

\section{REFERENCES}

[1] A. Stove, "Linear FMCW radar techniques," IEE Proceedings For Radar and Signal Processing, vol. 139, no. 5, pp. 343-350, 1992.

[2] C. Hu, Y. Liu, H. Meng, and X. Wang, "Randomized switched antenna array FMCW radar for automotive applications," IEEE Transactions on Vehicular Technology, vol. 63, no. 8, pp. 3624-3641, Oct 2014.

[3] P. Brennan, L. Lok, K. Nicholls, and H. Corr, "Phase-sensitive FMCW radar system for high-precision antarctic ice shelf profile monitoring," IET Radar, Sonar \& Navigation, vol. 8, no. 7, pp. 776-786, Aug 2014.

[4] D. Gray, M. Viola, W. Moran, S. Samarasekera, P. May, B. Bates, K. Venkataraman, C. McCarroll, B. Ferguson, and D. McLaughlin, "WREN: A weather radar experimental network," in International Conference on Electromagnetics in Advanced Applications (ICEAA), 2010, Sept 2010, pp. 505-508.

[5] N. J. Willis and H. D. Griffiths, Eds., Advances in bistatic radar. SciTech Publishing, 2007.

[6] P. Stinco, M. Greco, F. Gini, and M. Manna, "Non-cooperative target recognition in multistatic radar systems," IET Radar, Sonar \& Navigation, vol. 8, no. 4, pp. 396-405, April 2014.

[7] T. Derham, S. Doughty, C. Baker, and K. Woodbridge, "Ambiguity functions for spatially coherent and incoherent multistatic radar," IEEE Transactions on Aerospace and Electronic Systems, vol. 46, no. 1, pp. 230-245, Jan 2010

[8] T. Derham, S. Doughty, K. Woodbridge, and C. Baker, "Design and evaluation of a low-cost multistatic netted radar system," IET Radar, Sonar \& Navigation, vol. 1, no. 5, pp. 362-368, October 2007.

[9] P. Howland, D. Maksimiuk, and G. Reitsma, "FM radio based bistatic radar," IEE Proceedings on Radar, Sonar and Navigation, vol. 152, no. 3 , pp. 107-115, June 2005.

[10] K. Chetty, G. Smith, and K. Woodbridge, "Through-the-wall sensing of personnel using passive bistatic WiFi radar at standoff distances," IEEE Transactions on Geoscience and Remote Sensing, vol. 50, no. 4, pp. 1218-1226, April 2012.

[11] J. Brown, K. Woodbridge, H. Griffiths, A. Stove, and S. Watts, "Passive bistatic radar experiments from an airborne platform," IEEE Aerospace and Electronic Systems Magazine, vol. 27, no. 11, pp. 50-55, November 2012.

[12] F. Colone, D. O'Hagan, P. Lombardo, and C. Baker, "A multistage processing algorithm for disturbance removal and target detection in passive bistatic radar," IEEE Transactions on Aerospace and Electronic Systems, vol. 45, no. 2, pp. 698-722, April 2009.

[13] M. Malanowski and K. Kulpa, "Digital beamforming for passive coherent location radar,' in Radar Conference, 2008. RADAR 'O8. IEEE, May 2008, pp. 1-6.

[14] A. Hymans and J. Lait, "Analysis of a frequency-modulated continuouswave ranging system," Proceedings of the IEE - Part B: Electronic and Communication Engineering, vol. 107, no. 34, pp. 365 -372, July 1960.

[15] M. Skolnik, Introduction to Radar systems, 3rd ed. McGraw-Hill, 2001.

[16] W. B. Davenport, "Signal-to-noise ratios in bandpass limiters," Massachusetts Institute of Technology, Research Laboratory of Electronics, no. 234 , p. 18,1952

[17] K. Gentile, "Super-Nyquist operation of the AD9912 yields a high RF output signal," Analog Devices Application Note, 2007.

[18] M. Ash and P. V. Brennan, "Transmitter noise considerations in superNyquist FMCW radar design," IET Electronics Letters, vol. 51, no. 5, 2015.

[19] H. Rohling, "Radar CFAR Thresholding in Clutter and Multiple Target Situations," IEEE Transactions on Aerospace and Electronic Systems, no. 4, pp. 608-621, 1983. 